\title{
Curriculare Innovation im Spezialbereich: Qualifizierung im Themenbereich der Langzeitarchivierung digitaler Objekte
}

Heike Neuroth und Achim Oßwald

\section{Zusammenfassung}

Für das noch relativ neue Forschungs- und Praxisthema Langzeitarchivierung digitaler Objekte gibt es in Deutschland aus unterschiedlichen Gründen an noch keiner Hochschule ein umfassendes Lehrangebot. Im Rahmen des Projektes nestor hat eine länderübergreifende Kooperation von Hochschulen aus Deutschland, der Schweiz und Österreich ein breit gefächertes Qualifizierungsangebot realisiert, dessen Kern die Entwicklung von eLearning-Tutorials darstellt. Diese sollen als ein auf kooperativer Basis erstelltes, von allen beteiligten Hochschulen genutztes curriculares Angebot in die hochschulbasierte Aus-, Fort- und Weiterbildung integriert werden.

\section{Abstract}

As a relatively new topic in research and practice, long-term digital preservation is currently not very well covered by university curricula for a number of reasons. Project "nestor", a transnational cooperation of academic institutions in Germany, Switzerland, and Austria, has established a comprehensive programme based on elearning tutorials. These tutorials can be integrated into the standard curriculum at each institution.

\section{Rahmenbedingungen für die Entwicklung von Studiengängen}

Charakteristisch für die bislang in diesem Themenheft dargestellten Studien- und Qualifizierungsangebote ist der Umstand, dass sie

- von einer einzelnen Hochschule und

- mit dem Ziel einer fachlich breiten Qualifizierung oder - dem konsekutiven Ansatz folgend - einer fachlich affinen Vertiefung

- sowie unter Bezugnahme auf den sog. „Bologna-Prozess“

konzipiert wurden. Insbesondere mit der ersten Einflussgröße sind gewisse inhaltliche Nebeneffekte verbunden, deren Bedeutung für die Studierenden im curricularen Kontext meist erst im Nachhinein erkennbar und faktisch nicht beeinflussbar sind. Dies sind mindestens:

a) die Begrenzung der inhaltlichen Ausrichtung des Studienangebots abhängig von individuellen Schwerpunktsetzungen und der kapazitären Verfügbarkeit 
von Lehrenden sowie nicht zuletzt von Sichten, die in der Historie und kollektiven Orientierung des Lehrkörpers bzw. der Institution liegen;

b) marketingorientierte Abgrenzungen von Angeboten anderer Hochschulen;

C) das tatsächliche Aufgreifen von neuen, zukunftsrelevanten Themen und deren substantielle Integration in das Curriculum abhängig vom Innovationspotenzial der Lehrenden bzw. den anderen unter a) genannten Faktoren.

Beispiele für die unterschiedlich schnelle und fachlich ausdifferenzierte Diffusion von Themen in die Curricula der informationswissenschaftlichen Studiengänge aus den letzten zwei Jahrzehnten sind der Stellenwert der Informationstechnologie (konkret z.B. in den 80er und 90er Jahren des 20. Jahrhunderts die Automatisierung der Geschäftsgänge und IT-gestützte Datenhaltung, später dann die Einführung des Internet), die betriebswirtschaftlichen Betrachtung informationsorientierter Tätigkeiten (in den 90er Jahren - verbunden mit einem sich ausprägenden Kostenbewusstsein und Refinanzierungserwartungen) oder die Umorientierung von einer bestandsorientierten in eine angebots- und dienstleistungsorientierte Ausrichtung der Informationstätigkeiten (seit Mitte der 90er Jahre) bis hin zur Erprobung des Stellenwerts interaktiver und beteiligungsorientierter Optionen in jüngster Zeit.

Seit dem Beginn des sogenannten „Bologna-Prozesses“, ausgelöst durch die Erklärung der Bildungsminister von 29 europäischen Staaten am 19. Juni 1999 in Bologna über die Vereinheitlichung von Studienabschlüssen und Studiengangsstrukturen in der EU finden diese curricularen Neuerungen vor dem Hintergrund von politisch gewollten Angleichungen statt. Sie zeigen sich u.a. in Form einer Abschwächung der föderalen Unterschiede in den Angebotsstrukturen an deutschen Hochschulen. So erleichtert z.B. die Vereinheitlichung der Bewertung von Studienleistungen auf der Basis des European Credit Transfer System (ECTS) bislang nur erhoffte Formen der nationalen und internationalen Zusammenarbeit. Ein hochschulpolitisch interessanter Nebeneffekt aus bundesdeutscher Sicht ist dabei das Erodieren der - außerhalb des deutschsprachigen Raums ohnehin kaum kommunizierbaren - Differenzierung zwischen Universitäten und Fachhochschulen ${ }^{1}$.

\section{$2 \quad$ Langzeitarchivierung digitaler Objekte als neuer Gegenstand curricularer Planungen}

Eine ähnliche thematische Neuorientierung für den im weitesten Sinne informationswissenschaftlichen Bereich deutet sich mit der systematischen Umstellung des Publikationswesens auf digital basierte Prozesse und Produkte an. Dies beeinflusst methodisch nahezu alle Tätigkeitssegmente im bibliothekarischen und weiteren informationswirtschaftlichen Kontext. Faktisch bedeutet dies für die meisten bibliothekarischen Institutionen parallele Aufgabenbereiche und Prozesse, weil nur in wenigen Anwendungsbereichen die völlige Umstellung auf digitale Bestände und Dienstleistungen realisierbar und gewünscht ist. Eine Konsequenz ist die gestiegene Anzahl an sogenannten Universitätsverlagen (University Press), die 
zumeist auf Ebene einer Hochschule die digitalen Publikationen sammeln und herausgeben. ${ }^{2}$ So hat auch schon im Jahr 2002 die Hochschulrektorenkonferenz empfohlen, das wissenschaftliche Informations- und Publikationswesen in den nächsten Jahren konsequent auf elektronische Kommunikations- und Informationsmöglichkeiten auszurichten. ${ }^{3}$

Dies gilt auch für das Thema Bestandssicherung, das in der Konsequenz in Bibliotheken zweigleisige Aktivitäten fordert: Die für die traditionellen Printprodukte einerseits wie auch jene für die in den letzten Jahren in Zahl und Relevanz einen wachsenden Stellenwert erfahrenden digitalen Objekte andererseits.

Ginge es um Bibliotheken und ggf. die anderen Kulturerbeeinrichtungen allein, würde dieser Umstand vermutlich wenig Beachtung erfahren. Weil jedoch das

Bestandssicherungs-Know-how für digitale Objekte - in der öffentlichen (Fach)Diskussion als Langzeitarchivierung digitaler Objekte bezeichnet - aus wichtigen anderen gesellschaftlichen Segmenten, speziell dem der Wirtschaft, aber auch aus dem privaten Bereich nachgefragt wird, ist der Erwartungsdruck auf die Experten in Bibliotheken, Archive und Museen zur Bereitstellung spezifischer Problemlösungen sehr rasch und deutlich gestiegen - und damit automatisch auch der Erwartungsdruck auf die Hochschuleinrichtungen, die für diese Arbeitsmarktsegmente qualifizieren.

Wie eingangs dargelegt, war und ist es eher von intrinsischen Faktoren abhängig, ob das Thema „Langzeitarchivierung digitaler Objekte“ von einzelnen Lehrenden an einer Hochschule aufgegriffen wird. ${ }^{4}$ Vor diesem Hintergrund erfolgte eine curriculare Einbindung dieses Themas zum Beispiel mit den Schwerpunkten im Organisatorischen (Strategie, Sammlungsschwerpunkte etc.), Finanziellen (Geschäftsmodelle, Finanzierung etc.) oder Technologischen (Archivsysteme, IngestProzesse etc.) bei der Qualifizierung von Bibliothekarinnen und Bibliothekaren, aber auch von Mitarbeiterinnen und Mitarbeitern anderer sog. Kulturerbeeinrichtungen wie z.B. Museen bislang nur sehr begrenzt.

Die curriculare Situation stellt sich derzeit wie folgt dar:

- In einzelnen Lehrveranstaltungen erfolgte eine thematische Einführung und Bestandsaufnahme und insofern eine eher punktuelle Qualifizierung (vgl. Oßwald / Scheffel 2007).

- Auch das in Deutschland bislang am deutlichsten auf den Bereich der Langzeitarchivierung digitaler Objekte ausgerichtete Qualifizierungsangebot, das MA-Studienangebot der Kunstakademie Stuttgart (http://www.mediaconservation.abk-stuttgart.de/) fokussiert auf einen speziellen Anwendungsbereich.

- Als neuer Themenschwerpunkt und eventuelles zukünftiges Lehrgebiet einer darauf spezialisierten Professur könnte es in den neuen BachelorStudiengangskonzepten nur bei großem Problemdruck und unter Verzicht auf ein anderes, traditionelles oder ggf. ebenfalls neues Thema in das Curriculum integriert werden - und dies auch frühestens beim nächsten anstehenden 
Studienreformzyklus (alle 5-7 Jahre). ${ }^{5}$ Hinzu kommt, dass das Thema Langzeitarchivierung digitaler Objekte ein typisches Vertiefungsthema und insofern vorzugsweise für einen Master-Studiengang geeignet ist.

- Für die Realisierung eines MA-Angebotes fehlen sowohl die personellen Ressourcen (im Sinne von Wissenschaftlern mit entsprechenden theoretischem und berufspraktischem Hintergrund, die die für eine solches Angebot notwendige breite fachliche Qualifikation und Lehrerfahrung mitbringen) als auch stabile arbeitsmarktbezogene Prognosen, die das Auslastungs- und damit auch Finanzierungsrisiko eines solchen Einzelangebotes für eine Hochschule allein rechtfertigen würden. ${ }^{6}$

Vor diesem Hintergrund eröffnet sich die Option - je nach fachlich verantwortlicher Sicht aber auch die Notwendigkeit - zu neuen Formen der Zusammenarbeit von Hochschuleinrichtungen und außerhochschulischen Qualifizierungseinrichtungen zu kommen, die die Relevanz des Themas für ihre jeweilige Klientel sehen - und das Aufgreifen des Themas auch als Indiz für ihre Innovationsoffenheit wahrgenommen wissen wollen.

\section{Hochschulübergreifende Kooperationen}

\subsection{Zielsetzungen der hochschulübergreifenden Zusammenarbeit}

Am Fallbeispiel der im Rahmen von nestor projektbasiert erfolgten Zusammenarbeit verschiedener Hochschulen und anderer fachlich einschlägiger Qualifizierungseinrichtungen deutet sich hier ein neues Modell curricularer Kooperation an, bei dem aus der (kapazitären und spezialisierungsbezogenen) Not eine Tugend (mit kooperativen und kollaborativen Innovationspotenzialen) erwachsen könnte.

Die Kooperation zwischen nestor und den Hochschulen bzw. anderen Qualifizierungseinrichtungen wird dabei von dem nestor-Partner Staats- und Universitätsbibliothek Göttingen als zentralen thematischen Ansprechpartner koordiniert. Modellhaft ist dabei nicht nur der Prozess und die Form der Zusammenarbeit, sondern auch die Zielsetzung, ein neues und hochrelevantes Thema unter den skizzierten hochschulrechtlichen Rahmenbedingungen im Interesse der Fachwelt nicht konkurrierend, sondern kooperativ aufzugreifen.

Schritte auf dem Weg zu diesem Ziel sind nachfolgend skizzierten Formen der Zusammenarbeit im Sinne einer gestufte Entwicklung von

- kooperativer Zusammenarbeit bei der Konzeption und Realisierung von Fortbildungsveranstaltungen;

- gemeinsam genutzten Lehr- und Lernmaterialien;

- kooperativ und nach gemeinsam vereinbarten Standards entwickelten eTutorials; 
- der gegenseitigen Anerkennung von fachlich einschlägigen Lehrmodulen (z.B. auf der Grundlage von ECTS Punkten) bis hin zu einem

- kooperativ konzipierten und realisierten Studienschwerpunkt bzw. Studienangebot.

\section{Exkurs:}

\section{nestor und seine Aktivitäten im Bereich Langzeitarchivierung und Langzeitverfügbarkeit digitaler Objekte}

nestor (www.langzeitarchivierung.de) ist das deutsche Kompetenznetzwerk zur digitalen Langzeitarchivierung. In nestor arbeiten Bibliotheken, Archive, Museen und führende Experten gemeinsam am Thema Langzeitarchivierung und Langzeitverfügbarkeit digitaler Quellen. nestor ist ein vom BMBF-gefördertes Projekt mit einer Gesamtlaufzeit von insgesamt 6 Jahren in zwei Förderphasen. Ziel ist der Aufbau einer dauerhaften Organisationsform für alle Belange der Langzeitarchivierung in Deutschland. Auch die Vernetzung und Kooperation mit nationalen und internationalen Partnern ist ein Kernthema von nestor.

Die Zielgruppen von nestor sind:

- Institutionen, zu deren Aufgaben die Archivierung und Langzeiterhaltung digitaler Ressourcen gehört

- Personen, die über Kompetenzen und Erfahrungen auf dem Gebiet verfügen

- Produzenten digitaler Ressourcen in Wissenschaft, Wirtschaft und Verwaltung

- Nutzer digitaler Ressourcen

- Förderinstitutionen mit deren Rahmenplanungen und Einzelaktivitäten

- ausländische Institutionen, Organisationen und Projekte, die auf dem Gebiet der Langzeitarchivierung digitaler Ressourcen aktiv sind

- kommerzielle Dienstleister und Industriepartner, die Services oder Produkte zur Langzeitarchivierung anbieten

Das Kompetenznetzwerk nestor verfolgt das Ziel, die digitalen Ressourcen in Deutschland zu sichern und verfügbar zu machen und mit anderen Netzwerken und Entscheidungsträgern national und international zusammenzuarbeiten, um gemeinsam das kulturelle und wissenschaftliche Erbe Deutschlands langfristig zu bewahren.

Verschiedene Arbeitsgruppen (vgl. http://nestor.cms.hu-berlin.de/moinwiki) unterstützen dabei derzeit die nestor-Aktivitäten:

- AG Vertrauenswürdige Archive - Zertifizierung

- AG Media

- AG Langzeitarchivierungsstandards

- AG kooperative Langzeitarchivierung

- AG Vernetzung Grid / eScience und Langzeitarchivierung 
- AG Kooperation mit Hochschulen im Bereich Aus-, Fort- und Weiterbildung

\subsection{Kooperationspartner bei der Entwicklung neuer Qualifizierungsangebote}

Insbesondere die Arbeitsgruppe „Kooperation mit Hochschulen im Bereich Aus-, Fort- und Weiterbildung“, die im Rahmen des nestor Arbeitspaketes 5 „Einrichtung und Ausbau von Ausbildungs- und Fortbildungsangeboten“ ins Leben gerufen wurde, konnte gänzliche neue Kooperationspartner für nestor gewinnen. Insgesamt acht Partner aus Hochschulen und Weiterbildungseinrichtungen aus Deutschland, Österreich und der Schweiz sind zurzeit in dieser Arbeitsgruppe engagiert:

- Niedersächsische Staats- und Universitätsbibliothek Göttingen (Leitung)

- Archivschule Marburg

- Fachhochschule Köln, Institut für Informationswissenschaft

- Humboldt University Berlin - Institut für Bibliotheks- und Informationswissenschaft (IBI)

- Hochschule für Technik, Wirtschaft und Kultur Leipzig, Fachbereich Medien

- Fachhochschule Potsdam, Fachbereich Informationswissenschaften

- Hochschule für Technik und Wirtschaft Chur (Schweiz), Informationswissenschaft

- Technische Universität Wien (Österreich), Information \& Software Engineering Group

Ausgehend von Vorarbeiten im Rahmen der ersten Phase von nestor wurden die Arbeitsschwerpunkte für die 2. Laufzeit des Projektes konzipiert. Dabei war von Beginn an auch vorgesehen, im Bereich von Lern- und Lehreinheiten zusammenzuarbeiten. Die nestor Seminare, die in nestor I entstanden sind und zum Teil per Videoaufzeichnung auf DVD auch für spätere Lehreinheiten nachgenutzt werden konnten, stellten eine Ausgangsbasis für weitere, engere Kooperationen dar.

\subsection{Ein „Memorandum of Understanding“ über die Zielsetzungen der Kooperationspartner}

Die Ziele der seit 2006 aktiven Arbeitsgruppe sind in einem gemeinsamen, im 2007 von den verantwortlichen Vertretern der Hochschulen unterzeichneten „Memorandum of Understanding“ (http://nestor.sub.unigoettingen.de/education/mou.pdf) festgehalten:

- Die Partner vereinbaren den wechselseitigen Informationsaustausch zu Fragen der Qualifizierung im Bereich der langfristigen digitalen Archivierung.

- Die Partner streben an, mittelfristig den curricularen Anteil des Themas digitale Langzeitarchivierung in der Lehre auszubauen, soweit bei ihnen ein entsprechendes Lehrangebot besteht. Die Lehrenden streben an, sich bei der gemeinsamen Entwicklung der Module auf Aspekte der digitalen 
Langzeitarchivierung zu spezialisieren und sich gegenseitig zu ergänzen. Dadurch kann eine Profilbildung der jeweiligen Institution stattfinden.

- Soweit bei innen ein entsprechendes Lehrangebot besteht, treiben die Partner perspektivisch die Entwicklung eines gemeinsamen Curriculums voran, das zwischen den Hochschulen in verteilten, unterschiedlichen Schwerpunkten (wie z. B. Technik, Organisation, Standards etc.) angeboten und genutzt werden kann. Hierzu kann auch die Entsendung von Dozenten oder Studierenden zählen. Eine Möglichkeit der Realisierung könnte ein gemeinsames, modular angebotenes MA-Studium sein, soweit die entsprechenden Voraussetzungen an den Partnerinstitutionen gegeben sind.

- Alle beteiligten Partner werden Materialien in das curriculare Konzept einbringen, die von den hier genannten Partnern inhaltlich geprüft und ggf. mit einem Zertifikat versehen werden können.

- In Abhängigkeit von den institutionellen Rahmenbedingungen ist die gegenseitige Anerkennung von Lehrveranstaltungen und der damit erworbenen ECTS Punkte beabsichtigt.

Dieses Memorandum of Understanding hat zunächst eine Laufzeit, die an die Projektlaufzeit von nestor gekoppelt ist. Bereits jetzt werden aber erste Diskussionen geführt, wie die Kooperation und Kollaboration nach Ende des BMB-Projektes nestor aussehen kann. Zu diesen Überlegungen gehört auch die Frage, ob und welche weiteren Partner für diese Form der Zusammenarbeit gewonnen werden können.

\section{Vorcurriculare Qualifizierungsangebote im Themenbereich der digitalen Langzeitarchivierung}

Im Projekt nestor wurde ein umfangreicher Qualifizierungsbedarf erkannt, der bis zur Verankerung des Themas in der Hochschulausbildung über Weiterbildungsangebote bedient werden muss. Daher wurde damit begonnen, ein Konzept für ein Fort- und Weiterbildungsangebot in Zusammenarbeit mit bestehenden Qualifizierungseinrichtungen aus dem Bereich Bibliothek, Archiv und Museum zu entwerfen.

Diese Aktivitäten wurden konkret in nestor II mit der nestor Spring School 2007 eröffnet. Die Reihe der mehrtägigen Training Schools wurde und wird mit ähnlichen Veranstaltungen fortgesetzt, aber auch andere Veranstaltungen wie Workshops, Tagungen, Seminare etc. werden angeboten.

Ein weiterer Baustein der nestor Bemühungen um eine umfassende Qualifizierung ist das "nestor-Handbuch: Eine kleine Enzyklopädie der digitalen Langzeitarchivierung" das im Frühjahr 2007 in einer ersten Version der Öffentlichkeit vorgestellt wurde. Zukünftig wird dieses Handbuch in weiteren überarbeiteten und ergänzten Versionen veröffentlicht werden.

Als weiteres Angebot erfolgte die Entwicklung von ergänzenden e-Learning-Modulen. Im Folgenden werden die einzelnen Bausteine vorgestellt: 


\section{1 nestor Schools}

Nach dem Vorbild der Delos Summer Schools ${ }^{7}$ und den ab 2008 startenden Digital Preservation Europe (DPE) Schools (http://www.digitalpreservationeurope.eu/) sind im Jahr 2007 zwei nestor Schools veranstaltet worden, die nestor Spring School (http://nestor.sub.uni-goettingen.de/spring school 2007/index.php) und die nestor Winter School (http://nestor.sub.uni-goettingen.de/winter school 2007/index.php). Mit jeweils über 40 Teilnehmern und Referenten waren die Schools sehr erfolgreich, zumal ein hoher Prozentsatz an Studierenden gewonnen werden konnte.

Das Konzept der Schools besteht in der Zusammensetzung unterschiedlicher theoretischer und praktischer Blöcke. Jeweils eine 1,5-stündige Lektion führt in das Thema ein (z.B. Metadaten, Formate, Vertrauenswürdige Archive), danach folgt eine praktische Übung in mehreren kleinen Gruppen, die von den Referenten und Experten intensiv betreut wird. Die Teilnehmer stellen die Ergebnisse ihrer Übung dem Plenum vor und zum Abschluss wird das Themengebiet zusammenfassend diskutiert. Da die Schools jeweils für eine Woche angelegt sind, konnte eine umfassende Einführung in das Thema der digitalen Langzeitarchivierung (nestor Spring School) gegeben bzw. im Rahmen der Winter School der Fokus auf praktische Anwendungsfelder gelegt werden.

Mit zum Konzept gehört, dass die Referentinnen und Referenten nicht nur möglichst während der gesamten Veranstaltung anwesend sind, sondern sich auch als Spezialisten bei Übungen und Gesprächen über die Praxiserfahrung der Teilnehmerinnen und Teilnehmer mit ihrer Expertise einbringen und so zusätzlich die Lernprozesse vertiefen.

\subsection{Das nestor-Handbuch}

Die Langzeitarchivierung digitaler Objekte gewinnt sowohl national als auch international zunehmend an Bedeutung. Das „nestor Handbuch: Eine kleine Enzyklopädie der digitalen Langzeitarchivierung" (http://nestor.sub.unigoettingen.de/handbuch/) versucht, das derzeit vorhandene Wissen über das vielfältige und komplexe Thema und seine unterschiedlichen Teilaspekte zu sammeln und über eine "kleine Enzyklopädie" in strukturierter Form einer deutschsprachigen Gemeinschaft zugänglich zu machen.

Einzelne, von verschiedenen Experten erstellte Fachaufsätze gestatten einen Einblick in die diversen Themengebiete der Langzeitarchivierung: von technischen und rechtlichen Aspekten bis hin zur Definition von Rahmenbedingungen.

Das nestor Handbuch wird als "living document" verstanden, in dessen vorliegender Version 1.2 schon eine Reihe von Themen gesammelt werden konnten. Die Beiträge des Handbuchs werden im Laufe der Zeit ergänzt, vervollständigt und aktualisiert.

Damit verbunden ist auch, dass der Kreis der Experten laufend erweitert und ergänzt wird. Ziel ist dabei in naher Zukunft einen umfassenden Überblick über das 
anspruchsvolle und sich in stetiger Entwicklung befindliche Themengebiet zu erhalten.

Angeboten wird auch die Möglichkeit, die deutschsprachige Fachgemeinschaft in den Entstehungsprozess einzubeziehen, indem Kommentare ${ }^{8} z u$ den einzelnen Artikeln in das technische System eingestellt werden können. ${ }^{9}$

\subsection{Seminare}

Bisher wurden mehrere, meistens eintägige, Seminare zu bestimmten Themen angeboten, die durchweg sehr gut besucht waren. Der Vorteil der Seminare ist, dass schnell und unkompliziert auf aktuelle Bedürfnisse der LangzeitarchivierungsCommunity reagiert und in dieser Form relativ unaufwändig Unterstützung und Hilfe angeboten werden kann. Die Seminare spezialisieren sich in der Regel auf bestimmte Themenbereiche und haben nicht unbedingt immer nur einen einführenden und grundsätzlichen Charakter.

\section{4 eLearning-Tutorials}

Studierende aus den Fachhochschulen Köln, Potsdam und Leipzig sowie der Hochschule für Technik und Wirtschaft Chur in der Ostschweiz beteiligten sich im Wintersemester 2007/2008 an einem gemeinsamen Projekt zur Entwicklung von eLearning-Tutorials zu verschiedenen Themenfeldern der Langzeitarchivierung digitaler Objekte. Die Tutorials bieten einführende und inhaltlich vertiefende Informationen, die unter Nutzung der auch international weit verbreiteten e-Learning Plattform Moodle (http://www.moodle.de) entwickelt wurden. Diese SoftwareAnwendung wird von der Humboldt-Universität zu Berlin (HUB) technisch bereit gestellt und betreut. Außerdem wurde von der HUB ein gestalterisches und didaktisches Konzept für die e-Learning-Tutorials entworfen.

Auf der Grundlage von vier Seminar- bzw. Projekt-Veranstaltungen, die von Hochschullehrern ${ }^{10}$ an den jeweiligen Standorten initiiert, koordiniert und betreut wurden, bereiteten die Studierenden z.B. folgende Themen in Form von e-LearningTutorials auf:

- Einführung in die Langzeitarchivierung digitaler Objekte

- Formate und Datenträger in der Langzeitarchivierung

- Langzeitarchivierung bestimmter Datentypen (CAD-Daten, GIS-Daten)

- Metadatenerzeugung für technische Abläufe in der Langzeitarchivierung (z.B. ingest)

In den kommenden Semestern werden diese Tutorials an verschiedenen Hochschulstandorten in Lehrveranstaltungen evaluiert und optimiert. Auch ist geplant, von Studierenden weitere Module im Rahmen hochschulübergreifender Seminare entwickeln zu lassen, die an den jeweiligen Hochschulen von gemeinsamen Projektveranstaltungen begleitet werden. 


\section{Curriculare Optionen für eine Integration in die hochschulbasierte Aus-, Fort- und Weiterbildung}

Auf Grundlage der bislang erfolgten Aktivitäten und Erfahrungen in der Zusammenarbeit wird das im „Memorandum of Understanding“ formulierte Ziel

„Soweit bei ihnen ein entsprechendes Lehrangebot besteht, treiben die Partner perspektivisch die Entwicklung eines gemeinsamen Curriculums voran, das zwischen den Hochschulen in verteilten, unterschiedlichen Schwerpunkten (wie z. B. Technik, Organisation, Standards etc.) angeboten und genutzt werden kann. Hierzu kann auch die Entsendung von Dozenten oder Studierenden zählen. Eine Möglichkeit der Realisierung könnte ein gemeinsames, modular angebotenes MA-Studium sein, soweit die entsprechenden Voraussetzungen an den Partnerinstitutionen gegeben sind." (http://nestor.sub.unigoettingen.de/education/mou.pdf)

der kooperativen Entwicklung eines gemeinsam und modular konzipierten MAStudienangebotes immer realistischer erreichbar. Hierzu hat schon bislang die Entwicklung der eTutorials und die darin zum Ausdruck kommende thematische Schwerpunktsetzung beigetragen, wobei hiermit für die Beteiligten keine kompetenzbezogene Festlegung oder gar Einschränkung verbunden sein muss. Den einzelnen, jetzt schon beteiligten oder zukünftig hinzu kommenden Lehrenden und Hochschuleinrichtungen verbliebe weiterhin die Option, individuelle Kompetenzbereiche oder standortbezogene Forschungsschwerpunkte in dieses offene Konzept einzubringen. Damit ist auch weiterhin die Profilbildung jeder kooperierenden Hochschule sicher gestellt.

Mit der stabilen Bereitstellung und Pflege der e-Learning-Tutorials bestünde für die beteiligten Hochschulen, ihre Lehrende sowie die beteiligten kompetenten Praktiker, eine gemäß den Anforderungen der Kultusministerkonferenz ${ }^{11}$ zertifikatsbasierte Zusatzqualifikation anzubieten, die solitär oder z.B. als Erweiterung der ohnehin angebotenen berufsbegleitenden Master-Fernstudiengänge in Berlin und Köln zertifiziert realisiert werden könnte. Hierfür bedarf es lediglich der Bereitschaft, die bislang schon begonnenen, von nestor initiierten Aktivitäten konsequent weiter zu führen und in ein qualitativ abgesichertes, hochschulübergreifendes Konzept einzubringen.

Damit wäre es den beteiligten Hochschulen und den anderen Qualifizierungseinrichtungen möglich, auf der Grundlage fachlicher Zusammenarbeit im Interesse der Fachcommunity und deren Entwicklung über ihren wettbewerbsorientierten „Schatten“ zu springen und anstelle traditioneller, zu Zersplitterung der Ressourcen und Kompetenzen führenden Konkurrenz zukunftsorientierte Formen der Zusammenarbeit zu finden, die auch für andere Bereiche wegweisend sein könnten. 


\section{$6 \quad$ Kooperationsmöglichkeiten für die weiteren Entwicklungsschritte}

Es gibt vielfältige Möglichkeiten der Kooperation, die in Zukunft - auch über das Projektende von nestor hinaus - mit Blick auf ein curricular eingebundenes Qualifizierungsangebot verfolgt werden können.

In den USA läuft zum Beispiel bis 2009 das für die diese Perspektive hochinteressante Projekt, "DigCCurr - Preserving Access to Our Digital Future: Building an International Digital Curation Curriculum" (http://www.ils.unc.edu/digccurr), an dem unter Federführung der School of Information and Library Science (SILS) der Universität von North Carolina / Chapel Hill ein „openly accessible graduate-level curriculum“ (ebd.) entwickelt wird (vgl. Lee 2007). Erste Ergebnisse zeigen, dass hier versucht wird, das Thema der digitalen Langzeitarchivierung umfassend in allen Ebenen eines auf den Bereich digitaler Bibliotheksaufgaben orientierten Curriculums mit zu denken und entsprechend zu berücksichtigen. Digitale Langzeitarchivierung wird verstanden als ein Prozess, der den kompletten Lebenszyklus eines digitalen Objektes umfasst (digital curation). Dementsprechend sind in einer ersten, unveröffentlichten Version neun von zehn Kernthemen, die für die Qualifizierung im Bereich Digitale Bibliothek identifiziert wurden, langzeitarchivierungsrelevante Themenaspekte zugeordnet (z. B. für den Themenbereich „Digital Objects“ u.a. der Aspekt „2-c File formats, transformation, migration“). Ein Themencluster nennt sich sogar explizit „Preservation“. Interessant an diesem Ansatz ist, dass das Forschungs- und Lehrgebiet der digitalen Langzeitarchivierung komplett in das ganze Studienprogramm integriert werden soll. Das Projekt wird in den USA mit anderen Hochschulen gemeinsam durchgeführt, so dass die Chance besteht, die integrative Sicht des Digital Curation auch an anderen Studienstandorten einzubringen.

Die sich daran anschließende Frage in diesem Projektkontext ist, wie ein adäquater Studienabschluss aussehen könnte. Diskutiert werden, wie in Deutschland auch, verschiedene Ansätze wie zum Beispiel Informationsspezialist mit Schwerpunkt digitale Langzeitarchivierung. Sicherlich wäre hilfreich, hier in Kooperation mit allen relevanten Partnern national, aber auch international, zu gemeinsamen Konzepten zu kommen.

Eine weitere Gestaltungsvariante im nestor Kooperationsrahmen besteht darin, mit Hilfe englischsprachiger internationaler Partner, die eTutorials in die englische Sprache zu übersetzen, sie damit zu „internationalisieren“ und hierbei auch zu standardisieren. Gerade im Hinblick auf den Bologna Prozess könnte hiermit ein Grundstock gelegt werden, um sich zumindest auf europäischer Ebene enger zu vernetzen. Es besteht jedoch auch Interesse bei Partnern in außereuropäischen Ländern. Dies könnte in Zukunft zu einer Zusammenarbeit auf Hochschulebene führen, im Rahmen derer Studierende einen Teil ihres Studiums an einer deutschsprachigen und einen weiteren Teil an einer anderen europäischen Hochschule absolvieren. Voraussetzung hierfür wäre die gemeinsame Abstimmung über relevante Lehrinhalte sowie die gegenseitige Anerkennung von ECTS Punkten. 
Dies wäre bei der gemeinsamen Entwicklung von eTutorials fast automatisch gegeben.

Darüber hinaus bietet eine umfangreiche, qualitätsgeprüfte, z.B. nestor-zertifizierte Sammlung von eTutorials natürlich die Möglichkeit, auf individuell oder institutionell abgestimmte Bedürfnisse, eine Qualifizierungsmaßnahme zusammenzustellen Einzelne e-Tutorial Module könnten für bestimmte Anforderungen zusammengestellt wurden, so dass externe Schulungs- und Fortbildungsveranstaltungen im Hochschulbereich, aber auch bei den Gedächnisorganisationen (Bibliotheken, Archive, Museen) oder Industrievertretern angeboten werden könnten. Der charmante Nebeneffekt könnte darin bestehen, dass hierüber Einkünfte erzielt werden könnten, die wiederum z.B. in den Ausbau der technischen Plattform oder in den Erwerb von kostenpflichtigen Tools zur Erstellung von Lehr- und Lernmodulen investiert werden könnten. Ein solches Angebotskonzept böte gleichzeitig die Chance, die durch verschiedene Förderprogramme des BMBF (vgl z.B. http://www.bmbf.de/foerderungen/12128.php) oder der Europäischen Kommission ${ }^{12}$ signalisierte Zielvorstellung lebenslangen Lernens zu bedienen, die für das Bestehen in unserer heutigen Wissensgesellschaft unabdingbar ist.

Diese Vorstellung scheint sich auch mit den derzeitigen Entwicklungen und Diskussionen in Deutschland zu decken, die der Lehre im Hochschulbereich einen höheren Stellenwert verschaffen möchte. ${ }^{13}$ Auch wenn im Gegensatz zu der Exzellenzinitiative für die Forschung gesamt 1,9 Milliarden Euro zur Verfügung standen und in der zurzeit diskutierten (Stifterverband, KMK) „Exzellenzinitiative für die Lehre" nur ganze 5 Millionen Euro geplant sind, so könnte hiermit einer höherer Stellenwert der Lehre der Weg bereitet werden. Die Grundzüge der drei Förderlinien zeichnen sich nach dem derzeitigen Stand wie folgt ab:

- „Nachwuchsförderung“: Stipendien, Weiterbildungsangebote, Berufung von Gastprofessoren

- „Strukturbildung“: Kompetenzzentren für die Lehre inklusive Weiterbildung der Lehrenden

- „Strategieentwicklung“: Entwicklung von Zukunftskonzepten für die Angliederung an internationales Spitzenniveau im Bereich der Lehre

Die Gründung einer "Deutschen Lehrgemeinschaft" (vgl. z.B. http://idwonline.de/pages/de/news243140) analog zur Deutschen Forschungsgemeinschaft erscheint hier nur ein nächster konsequenter Schritt zu sein. Im Rahmen einer solchen Entwicklung könnte sich für die Hochschullandschaft in Deutschland ein interessantes Entwicklungspotential zeigen: Fachhochschulen und Universitäten könnten sich gemeinsam im Bereich der Lehre bzw. der kooperativen Hochschullehre engagieren. Dafür könnten die gemeinsamen Erfahrungen der nestor-Kooperation im Bereich der digitalen Langzeitarchivierung hilfreich, vielleicht sogar wegweisend sein.

\section{Zitierte Quellen und Literatur}


Lee, Christopher: International Digital Curation Curriculum: DigCCurr Project. Folien des Vortrags bei "iPRES 2007 - International Conference on Preservation of Digital Objects"; Beijing, 11-12 October, 2007 http://ipres.las.ac.cn/pdf/ipres2007-digccurr.pdf

Oßwald, Achim; Scheffel, Regine: Lernen und weitergeben - Aus- und

Weiterbildungsangebote zur Langzeitarchivierung

In: nestor - Handbuch - Eine kleine Enzyklopädie der digitalen Langzeitarchivierung: Version 0.1, 2007; S. 119-126 http://nestor.sub.uni-goettingen.de/handbuch/nestor-Handbuch 01.pdf

1 Vgl. dazu auch einen Artikel in der Süddeutschen Zeitung (4. Februar 2008) von Georg Krausch (Präsident der Johannes-Gutenberg-Universität in Mainz), in dem es unter anderem heißt: „Die deutsche Hochschullandschaft erlebt derzeit einen Umbruch, der seinesgleichen sucht in der jüngeren Geschichte: Die Umstellung des gesamten Lehrangebots im Zuge des Bologna-Prozesses, eine nie gekannte Konkurrenz zwischen den Universitäten, eine politisch gewollte Neudefinition des Verhältnisses zwischen Universitäten und Fachhochschulen und der Abbau der „Versäulung“ des deutschen Wissenschaftssystems sind nur einige Stichworte der gegenwärtigen Debatte."

Vgl auch Arbeitsgemeinschaft deutscher Universitätsverlage, http://www.ubka.unikarlsruhe.de/portale/ag_univerlage/

http://www.hrk.de/de/beschluesse/109_247.php?datum=198.+Plenum+am+5.+ November+2002

Selbst wenn die fachliche Weitsicht und Innovationsorientierung bei den zuständigen Leitungsgremien oder -personen gegeben ist, so sind hier erst jüngst begrenzte Steuerungsmöglichkeiten (W-Besoldung mit Zielvereinbarungen) eröffnet worden. Die traditionellen Beschäftigungsverhältnisse (Beamtenverhältnis auf Lebenszeit und damit geringe Erneuerungszyklen des Dozentenstammes) erlaubten so gut wie keine Steuerungsmöglichkeiten, sondern mussten weitgehend auf die intrinsisch motivierte Innovationsoffenheit der Lehrenden vertrauen.

Aus kapazitären Gründen ist dabei zumeist die Option auf Wahlpflichtangebote sehr reduziert. Inwieweit ein solches Wahlpflichtangebot von den Studierenden aufgegriffen würde, sei dahin gestellt.

Das Angebot der Kunstakademie Stuttgart (http://www.mediaconservation.abk-stuttgart.de/) bestätigt diese Einschätzung prinzipiell, weil es nur mit ungewöhnlich hoher finanzieller Förderung aus Landesmitteln realisiert werden konnte und zudem durch seine inhaltliche Ausrichtung auf eine sehr spezielle Zielgruppe fokussiert.

Vgl z.B Delos Summer School 2007, http://www.dpc.delos.info/ss07/index.php

Eine Testversion findet sich unter http://nestor.sub.uni-goettingen.de/handbuch_neu/index.php Inwieweit das Handbuch z.B. mit neueren Web 2.0 Technologien insgesamt interaktiver gestaltet werden kann und ob dies zu einer Qualitätssteigerung führt, muss im Laufe der Zeit sicherlich geprüft werden.

Prof. Dr. N. Stettler, HTW Chur; Prof. R. Scheffel, HTWK Leipzig; Dr. K. Schwarz, FH

Potsdam und Prof. Dr. A. Oßwald, FH Köln

11 Vgl. z.B. Ständige Akkreditierungskommission (SAK) - Arbeitsgruppe

Weiterbildungsstudiengänge: Verfahren und Standards zur Evaluierung und Akkreditierung von Weiterbildenden Studiengängen und Modulen (12.07.2005).

Vgl z.B. The Lifelong Learning Programme 2007-2013,

http://ec.europa.eu/education/programmes/newprog/index_en.html 